\title{
Immunohistochemical Profile of Breast Cancer Patients at a Tertiary Care Hospital in New Delhi, India
}

\author{
Dinesh Chandra Doval ${ }^{1,3 *}$, Anila Sharma², Rupal Sinha ${ }^{3}$, Kapil Kumar ${ }^{4}$, Ajay \\ Kumar Dewan ${ }^{4}$, Harit Chaturvedi ${ }^{5}$, Ullas Batra ${ }^{1}$, Vineet Talwar ${ }^{1}$, Sunil Kumar \\ Gupta $^{1}$, Shailendra Singh ${ }^{6}$, Vidula Bhole ${ }^{7}$, Anurag Mehta ${ }^{2}$
}

\begin{abstract}
Background: To assess the immunohistochemical expression of estrogen receptor (ER), progesterone receptor (PgR) and human epidermal growth factor receptor-2 (HER2) neu receptor in breast cancer and their associations with various clinicopathological characteristics. Materials and Methods: This is a retrospective analysis of women who presented with primary, unilateral breast cancer in the Department of Medical Oncology at Rajiv Gandhi Cancer Institute and Research Centre, Delhi, India during the period from January 2008 to December 2011. Data were retrieved from the medical records of the hospital including both early and locally advanced cancer cases. ER, PgR and HER2neu expression in these patients was assessed and triple negative patients were identified. Associations of triple negative and non-triple negative groups with clinicopathological characteristics were also evaluated. Results: A total of 1,284 women (mean age 52.1 years, $41.9 \%$ premenopausal) were included in the analysis. Hormone receptor positivity (ER and/or PgR) was seen in $63.4 \%$ patients, while $23.8 \%$ of tumors were triple negative. Only $23.0 \%$ were HER2 positive. Around $10.0 \%$ of tumors were both ER and HER2 positive. ER and PgR positivity was significantly associated with negative HER2 status (p-value $<0.0001$ ). Younger age, premenopausal status, higher tumor grade, lymph node negativity, advanced cancer stage, and type of tumor were strongly associated with triple negativity. Significantly, a smaller proportion of women had ductal carcinoma in situ in the triple negative group compared with the non-triple negative group $(35.6 \%$ versus $60.8 \%$, $p$-value $<0.01)$. Conclusions: The present analysis is one of the largest studies from India. The majority of the Indian breast cancer patients seen in our hospital present with ER and PgR positive tumors. The triple negative patients tended to be younger, premenopausal, and were associated with higher tumor grades, negative lymph nodes status and lower frequency of ductal carcinoma in situ.
\end{abstract}

Keywords: Breast cancer - immunohistochemistry - hormone receptors - India

Asian Pac J Cancer Prev, 16 (12), 4959-4964

\section{Introduction}

Breast cancer is the most common cancer among the females in terms of frequency \& mortality (Ferlay et al., 2010), and globally ranks 5th as a cause of cancer in general. Burden of breast cancer in India is high and increasing, especially in the urban areas (Ferlay et al., 2010). It was estimated that 145,000 Indian women were diagnosed with breast cancer and 70,000 died in the year 2012 (J Ferlay, 2012). The incidence of breast cancer is expected to increase from 153,297 cases to 235,490 cases during the period 2011 to 2026 (D'Souza et al., 2013).

Breast cancer is biologically heterogeneous, and the tumors show diversity of histopathological characteristics and clinical behavior (Munjal et al., 2009; Badve et al., 2011). Recently, the heterogeneity of hormonal receptors and their role in the clinical behavior of breast cancer has been identified.

The immunohistochemical staining for steroid hormone receptors (estrogen and progesterone receptors, ER and $\mathrm{PgR}$, respectively) and the human epidermal growth factor receptor-2 (HER2) helps to choose appropriate therapies and predict prognosis for breast cancer patients. For example, tamoxifen, a selective ER modulator, when used in patients with ER-positive breast cancer, leads to a better tumor response, longer survival, lower recurrence and reduced risk of death among patients with ER and/or PgR positive tumors than patients with ER and PgR negative tumors (Patil et al., 2011a). Trastuzumab, a humanized monoclonal antibody against the extracellular domain of HER2 and other targeted therapies has shown success in the outcome of patients with early and metastatic breast

${ }^{1}$ Department of Medical Oncology, ${ }^{2}$ Department of Laboratory Services, ${ }^{3}$ Department of Research, ${ }^{4}$ Department of Surgical Oncology, Rajiv Gandhi Cancer Institute \& Research Centre, ${ }^{5}$ Department of Surgical Oncology, MAX Hospital, ${ }^{6}$ Auriga Life Sciences, New Delhi, ${ }^{7}$ Episolutions Consultancy Services, Maharashtra, India *For correspondence: ddoval07@gmail.com 
cancer blocking downstream HER2 signaling to inhibit proliferation of tumor cells that overexpress HER2. However, it is not easily accessible to the patients due to its high cost and availability of generics has addressed this concern to some extent. Lack of expression of all three receptors (ER, PgR and HER2), the so called triple negative breast cancer (TNBC) is generally associated with poor prognosis (Basu et al., 2008).

Some data on immunohistochemistry (IHC) of breast cancer among Indian women are now available (Munjal et al., 2009; Vaidyanathan et al., 2010; Ambroise et al., 2011; Patil et al., 2011a; Dogra et al., 2014). However, HER2 staining for all newly diagnosed breast cancer patients is not a routine practice in many hospitals in India. Thus, data on all three IHC markers (ER, PgR and HER2) in Indian patients are scarce. Furthermore, previous published studies on IHC profile of breast cancer in Indian women had relatively small number of cases (Munjal et al., 2009; Vaidyanathan et al., 2010; Ambroise et al., 2011; Patil et al., 2011a). The present analysis was conducted to assess the IHC profile of breast cancer patients in the Indian population and further correlate the IHC expression with various clinico-pathological characteristics at presentation.

\section{Materials and Methods}

\section{Analysis participants}

This is a retrospective analysis of women who presented with primary, unilateral breast cancer in the Department of Medical Oncology at Rajiv Gandhi Cancer Institute and Research Centre, Delhi, India during the period from January 2008 to December 2011. Data was retrieved from the medical records of the hospital including both early and locally advanced cancer cases. Patients with metastatic breast cancer were excluded. Details of each patient related to the demographic profile, signs and symptoms, personal history, investigations, tumour profile, treatment and follow up were also recorded. The analysis was granted waiver by the Institutional Review Board of the Institute.

\section{Immunohistochemistry}

Tumor tissue samples (core biopsy/resection specimen) were fixed in $10 \%$ neutral buffered formalin for 12-24 hours. The appropriate sections of $1 \times 1 \mathrm{~cm}$ with a $3 \mathrm{~mm}$ thickness were processed in an autoprocessor for 6-48 hours (for HER2) or 6-72 hours (for ER, PgR) as per College of American Pathologist (CAP) guidelines and embedded in paraffin wax. Around $4 \mu \mathrm{m}$ thin sections were cut and spread onto the poly-L-lysine coated slides and dried overnight at room temperature. They were then deparaffinized using xylene and rehydrated with propanol.

Antigen retrieval for ER and PgR was done using TRIS EDTA buffer (pH9). The slide was immersed in a pressure cooker filled with heated TRIS EDTA buffer and brought to full pressure. Thereafter, the slide was cooled at room temperature, washed with tap water followed by washing in TRIS buffered saline (TBS). Then, the slide was dipped into $3 \% \mathrm{H}_{2} \mathrm{O}_{2}$ solution $(50 \mathrm{ml}$ Methanol +1.5 $\mathrm{ml} \mathrm{H}_{2} \mathrm{O}_{2}$ ) for 10 minutes to block endogenous peroxidase activity followed by 3 washes in TBS. Each slide was covered with $100 \mu 14 \%$ protein blocking reagent so as to block any non-specific antibodies. It was then incubated for one hour with 1:25 dilution of $100 \mu 1$ mouse anti ER monoclonal primary antibody (clone EP1, DAKO) anti PgR monoclonal primary antibody (clone PgR 636, DAKO). After 3 washes in TBS, the slide was treated with $100 \mu 1$ secondary antibody for $20 \mathrm{~min}$. It was again given 3 washes in TBS followed by incubation with $100 \mu \mathrm{l}$ polymer horse raddish peroxidase for $30 \mathrm{~min}$ at room temperature. The slide was again washed thrice in TBS. Thereafter, the slide was covered with freshly prepared DAB chromogen at room temperature for 10 minutes, washed in distilled water and counterstained with Gill's hematoxylin for 5 minutes. This was followed by dehydration with acetone and clearing by Xylene. The slide was then mounted with DPX, labelled and viewed under the microscope.

For HER2neu test, anti HER2neu primary antibody (HERCEPT, DAKO) was used. Antigen demasking was done by immersing the deparaffinized sections into pre heated citrate buffer ( $\mathrm{pH} 6$ ) in a coplin jar at a temperature of 90-950C in a water bath for $40 \mathrm{~min}$. Pressure cooker is not preferred for antigen demasking of HER-2. The slides along with the buffer was then brought to room temperature, and subsequently washed with tap water followed by three washings in TBS. The slides were dipped in $3 \% \mathrm{H}_{2} \mathrm{O}_{2}$ solution for 10 minutes to block endogenous peroxidase activity. Slide was then immersed in $100 \mu \mathrm{l}$ of $4 \%$ protein blocking reagent for $10 \mathrm{~min}$ at room temperature. This was followed by washing thrice in TBS and immersed in $100 \mu 1$ primary antibody for 1 hour at room temperature. The slides were rinsed gently in TBS three times and immersed in visualizing agent for $30 \mathrm{~min}$. Following this, the slide was incubated in $100 \mu \mathrm{l}$ DAB for $10 \mathrm{~min}$. It was washed with distilled water and counterstained with Gill's hematoxylin. This was followed by dehydration with acetone and clearing by Xylene. The

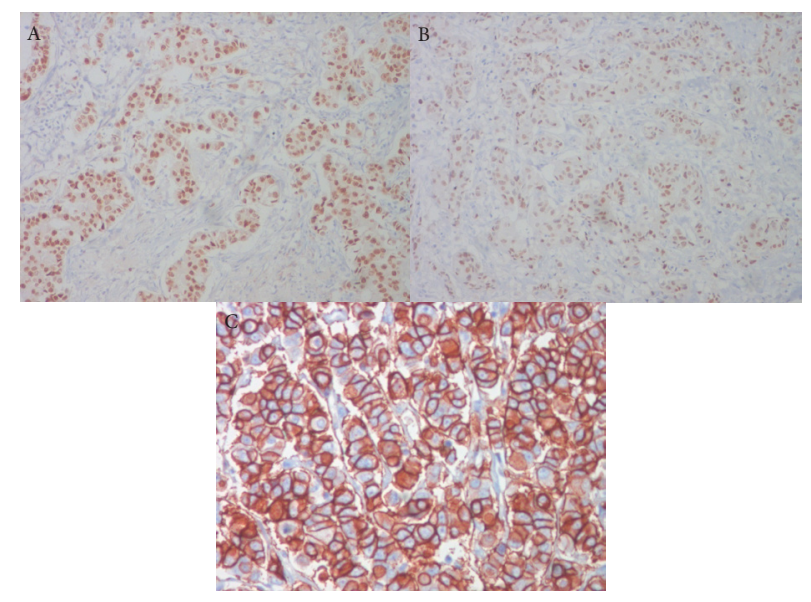

Figure 1.A: Estrogen Receptor (ER) Positive Slide with Strong Immunoexpression (Nuclear) in 70-80\% Tumor Cells (DAB; X100); B: Progesterone Receptor (PgR) Positive Slide Shows Moderate Immunoexpression (Nuclear) in 70-80\% Tumor Cells (DAB; X100); C: HER2 Positive Slide Shows Immunoexpression Score 3+ (DAB; X200) 
slide was then mounted with DPX, labeled and viewed under the microscope.

The stained sections were examined for appropriateness of staining of slide controls, batch controls and internal controls. After accessing the control slides/ internal control, the immunoreactivity of the test sample was scored as per the ASCO-CAP guidelines (2007) of ER/ PgR (Figure 1A and 1B) and HER2 (Figure 1C). Equivocal (HER2-2+) cases were tested for gene amplification by Fluorescent In-Situ Hybridization (FISH) as per FDA

Table 1. Clinicopathological features and Receptor Status (N=1284)

\begin{tabular}{|c|c|c|}
\hline Characteristic & Number & $\%$ \\
\hline \multicolumn{3}{|l|}{ Tumour grade (NBR) } \\
\hline 1 & 114 & 8.9 \\
\hline 2 & 600 & 46.7 \\
\hline 3 & 570 & 44.4 \\
\hline \multicolumn{3}{|c|}{ Tumour size (largest diameter) } \\
\hline$<20 \mathrm{~mm}$ & 144 & 11.2 \\
\hline$\geq 20 \mathrm{~mm}$ & 1140 & 88.8 \\
\hline \multicolumn{3}{|l|}{ Histopathology } \\
\hline IDC & 1216 & 94.7 \\
\hline Other* & 68 & 5.3 \\
\hline \multicolumn{3}{|l|}{ Lymph Node status } \\
\hline Positive & 679 & 52.9 \\
\hline Negative & 605 & 47.1 \\
\hline \multicolumn{3}{|l|}{ TNM Cancer stage } \\
\hline I & 112 & 8.7 \\
\hline II & 828 & 64.5 \\
\hline III & 344 & 26.8 \\
\hline \multicolumn{3}{|l|}{ ER Expression } \\
\hline Positive & 799 & 62.2 \\
\hline Negative & 485 & 37.8 \\
\hline \multicolumn{3}{|l|}{ PgR Expression } \\
\hline Positive & 683 & 53.2 \\
\hline Negative & 601 & 46.8 \\
\hline \multicolumn{3}{|l|}{ HER 2/neu status } \\
\hline Positive & 295 & 23 \\
\hline Negative & 989 & 77 \\
\hline \multicolumn{3}{|l|}{ ER / PR status } \\
\hline $\mathrm{ER}+\mathrm{PR}+$ & 668 & 52 \\
\hline ER+PR - & 131 & 10.2 \\
\hline ER-PR + & 15 & 1.2 \\
\hline ER-PR - & 470 & 36.6 \\
\hline \multicolumn{3}{|l|}{ Triple negative status } \\
\hline Triple negative & 305 & 23.8 \\
\hline Non-triple negative & 979 & 76.3 \\
\hline \multicolumn{3}{|c|}{$\begin{array}{l}\text { *HER2, human epidermal growth factor receptor 2; ER, estrogen } \\
\text { receptor; PgR, progesterone receptor; NBR, Modified Nottingham Bloom } \\
\text { Richardson scoring; IDC, infiltrating duct carcinoma; TNM, Tumor Node } \\
\text { Metastasis, * Lobular carcinoma in situ-5 }(0.39 \%) \text {, tubular-1 }(0.8 \%) \text {, } \\
\text { medullary-4 }(0.31 \%) \text {, mucinous-20 }(1.56 \%) \text {, papillary-2 }(0.16 \%) \text {, } \\
\text { cribriform-3 }(0.23 \%) \text {, invasive lobular carcinoma-33 }(2.57 \%)\end{array}$} \\
\hline
\end{tabular}

approved PATH VISION HER2 DNA Probe Kit II.

\section{Statistical analysis}

For continuous data, comparisons between groups were made using t-test. For categorical data, data analysis was conducted by using the Chi square test or Fisher's exact test as appropriate. Statistical analyses were conducted using GNU PSPP software (version 0.7.10). Statistical significance was evaluated at the 0.05 significance level for the two-sided tests.

\section{Results}

During the specified period of 4 years, a total of 1930 patients presented to the Medical Oncology Department with breast disease. Of these, a total of 646 patients were excluded from the analysis as shown in Figure 2. The present analysis included 1,284 women with early and locally advanced breast carcinoma with complete data availability.

Mean age of patients was 52.1 years (range 25-93 years). Almost half $(51.5 \%)$ of these patients were $<50$ years of age and $58.1 \%$ belonged to the post-menopausal group. A majority (82.1\%) of women underwent modified radical mastectomy (MRM). Around $48.8 \%$ patients had right sided breast cancer.

The data on clinico-pathological features and receptor status are tabulated in Table 1 . Around $94.7 \%$ patients had invasive ductal carcinoma on morphology and $88.8 \%$ tumors were $>20 \mathrm{~mm}$ in size. Modified Nottingham Bloom Richardson (NBR) scoring placed approximately $46.7 \%$ tumors in grade II. Metastases in axillary lymph nodes were found in $52.9 \%$ cases. Hormone receptor positivity

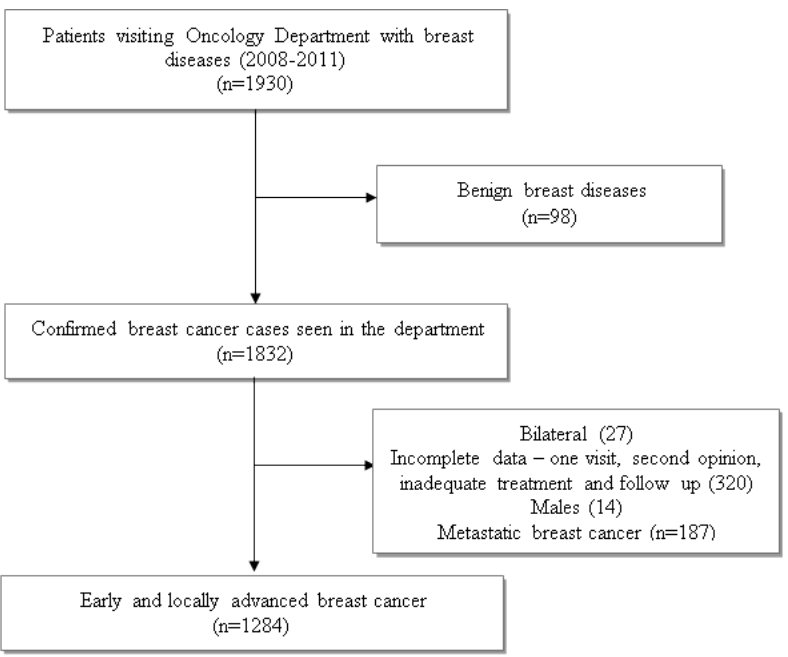

Figure 2. Flow of Participants

Table 2. The Association of HER2 Status with ER* and PR* Expression (N=1284)

\begin{tabular}{lccccc}
\hline HER2/neu status & ER+ & ER- & PgR+ & PgR- & Total \\
& $\mathrm{N}(\%)^{\$}$ & $\mathrm{~N}(\%)^{\$}$ & $\mathrm{~N}(\%)^{\$}$ & $\mathrm{~N}(\%)^{\$}$ & $\mathrm{~N}$ \\
\hline Positive & $128(43.4)$ & $167(56.6)$ & $94(31.9)$ & $201(68.1)$ & 295 \\
Negative & $671(67.9)$ & $318(32.2)$ & $589(59.6)$ & $400(40.4)$ & 989 \\
Total & $799(62.2)$ & $485(37.8)$ & $683(53.2)$ & $601(46.8)$ & 1284 \\
\hline
\end{tabular}

$* \mathrm{P}<0.0001 ;{ }^{\$}$ All percentages $(\%)$ are calculated row-wise, based on the row-total; Her-2, human epidermal growth factor receptor 2 ; ER, estrogen receptor; $\mathrm{PR}$, progesterone receptor 
Table 3. Characteristics of Triple-Negative Breast Cancers *

\begin{tabular}{|c|c|c|c|}
\hline Characteristic & $\begin{array}{l}\text { Triple negative } \\
\qquad(\mathrm{N}=305)\end{array}$ & $\begin{array}{l}\text { Non-triple negative } \\
\qquad(\mathrm{N}=979)\end{array}$ & $P$ value \\
\hline Age (mean $\pm S D$, years $)$ & $50.2 \pm 11.2$ & $52.7 \pm 11.2$ & $<0.01$ \\
\hline Age & & & 0.01 \\
\hline $20-50$ Years & $157(51.5)$ & $423(43.2)$ & \\
\hline $51-65$ Years & $122(40.0)$ & $427(43.6)$ & \\
\hline$>6$ Years & $26(8.5)$ & $129(13.2)$ & \\
\hline Menopause & & & $<0.01$ \\
\hline Premenopausal & $157(48.5)$ & $390(39.8)$ & \\
\hline Postmenopausal & $148(51.5)$ & $589(60.2)$ & \\
\hline Tumour grade (NBR) & & & $<0.01$ \\
\hline 1 & $7(2.3)$ & $107(10.9)$ & \\
\hline 2 & 75 (24.6) & $525(53.6)$ & \\
\hline 3 & $223(73.1)$ & $347(35.4)$ & \\
\hline Tumour size (largest diameter) & & & 0.09 \\
\hline$\leq 20 \mathrm{~mm}$ & $26(8.5)$ & $118(12.1)$ & \\
\hline$>20 \mathrm{~mm}$ & $279(91.5)$ & $861(88.0)$ & \\
\hline \multicolumn{4}{|l|}{ Lymph node status } \\
\hline Negative & $179(58.7)$ & $426(43.5)$ & $<0.01$ \\
\hline Positive & $126(41.3)$ & $553(56.5)$ & \\
\hline Cancer stage & & & $<0.01$ \\
\hline I & $23(7.5)$ & $89(9.1)$ & \\
\hline II & $221(72.5)$ & $607(62.0)$ & \\
\hline III & $61(20.0)$ & $283(28.9)$ & \\
\hline Type of tumour & & & $<0.01$ \\
\hline IDC & $300(98.4)$ & $916(93.6)$ & \\
\hline Other & $5(1.6)$ & $63(6.4)$ & \\
\hline Type of surgery & & & 0.06 \\
\hline MRM & $240(78.7)$ & $816(83.4)$ & \\
\hline $\mathrm{BCS}$ & $65(21.3)$ & $163(16.7)$ & \\
\hline DCIS & & & $<0.01$ \\
\hline Present & $103(35.6)$ & $567(60.8)$ & \\
\hline Absent & $186(64.4)$ & $365(39.2)$ & \\
\hline
\end{tabular}

SD, standard deviation; BCS, breast-conserving surgery; DCIS, ductal carcinoma in situ; IDC, infiltrating duct carcinoma; MRM, modified radical mastectomy; NBR, Modified Nottingham Bloom Richardson scoring; * Unless mentioned otherwise, the data are given as N (\%), the percentages are calculated column-wise

(for either ER and/or PgR) was seen in $63.4 \%$ of tumors. Only PgR positivity was shown by $1.17 \%$ tumors. Also, $23.8 \%$ tumors were triple negative.

Table 2 shows association of HER 2 status with ER and PgR expression. Only $23.0 \%$ of women had HER 2 positive status. HER 2 overexpression was seen in $17.3 \%$ of the hormone positive tumors and $28.6 \%$ of hormone negative tumors and hence a statistically significant association of HER2 negative status with ER and/or PgR positive tumors was observed ( $\mathrm{p}$-value $<0.0001$ ).

A total of $306(23.7 \%)$ patients were triple negative. The characteristics of TNBCs are highlighted in Table 3. Patients with triple negative tumors presented at a mean age of 50 years and majority of the patients $(51.5 \%)$ were in the age group of 20-50 years as compared to the non-triple negative tumor group ( $\mathrm{p}$-value 0.01 ). Triple negative tumors showed a strong statistical association with menopausal status ( $p$-value $<0.01$ ), tumor grade ( $p$-value $<0.01$ ), lymph node status ( $p$-value $<0.01$ ), cancer stage (p-value $<0.01$ ), type of tumor ( $p$-value $<0.01$ ), and ductal carcinoma in situ ( $\mathrm{p}$-value $<0.01)$. Premenopausal women $(51.5 \%)$, tumor grade $3(73.1 \%)$, tumor size $>20$ $\mathrm{mm}(91.5 \%)$, negative lymph node status $(58.7 \%)$, cancer stage II $(72.5 \%)$ and invasive ductal carcinoma (98.4\%) were more commonly observed among the patients with triple negative tumors.

\section{Discussion}

The present analysis assessed the relevance of IHC markers in relation to their clinico-pathological characteristics among 1,284 breast cancer patients in India. The analysis showed that $63.4 \%$ women were ER and/or PgR positive and only $23.1 \%$ women were HER2 positive. Overall, $23.7 \%$ women were triple negative and $10.0 \%$ tumors were both ER+/HER2 positive.

Hormone positivity is very important in determining the course of therapy as patients with positive hormonal status are suitable candidates for hormone therapy, given the higher numbers of low income strata, especially in developing countries like India (Manjunath et al., 2011). $\mathrm{PgR}$ mirrors the functional estrogenic stimulus and is a significant prognostic and predictive factor of the ER status (Patil et al., 2011a). We found 53.1\% PgR positive and $62.1 \%$ ER positive women in this analysis, which is very similar to results reported by Ambroise et al (Ambroise et al., 2011). Previous research suggests that about $45-65 \%$ Indian invasive breast cancer patients are positive for ER and/or PgR, and about $30-35 \%$ patients are negative for both ER and PgR (Ambroise et al., 2011; Patil et al., 2011a). The proportion of ER/PgR negativity was $36.7 \%$ and ER and/or PgR positivity was $63.4 \%$ in the present analysis. The proportion of $\mathrm{PgR}$ positive but 
ER negative tumors was $1.2 \%$, lower than that observed by Ambroise et al. (2011) and Patil et al. (2011a), but comparable to the analysis by Shet et al. (2009). This may be attributed to the clone which was being used for detecting progesterone receptor.

Our analysis found a significant inverse association between HER2 expression and ER/PgR expression (p-value $<0.0001$ ), though the proportion of HER2 positive tumors expressing ER (43.3\%) and PgR (31.9\%) was substantially high. Our results are in conformity with previous reports that HER2 amplification or overexpression is generally present in $20-30 \%$ of the breast cancer patients (Ambroise et al., 2011; Singhai et al., 2011) as $23.0 \%$ of our patients showed HER2 positivity. Our finding of $23.7 \%$ patients with triple negative status is similar to previous reports of $20-25 \%$ triple negative patients among Indian women with breast cancer (Ambroise et al., 2011; Patil et al., 2011b). The triple negative tumors were commonly grade III tumors. This is in line with similar results from other studies in India (Ambroise et al., 2011; Patil et al., 2011b).

Given the lack of awareness about screening, costs, fear and stigma associated with breast cancer in India, many women are diagnosed in later stages (Babu et al., 2013). The prognosis depends up to a great extent on various factors including metastatic disease, tumor size and grade, hormone status and HER2 status. Younger age, higher histopathological grade are characteristics of TNBCs (Patil et al., 2011b). Our analysis confirmed that triple negative women tended to be younger, premenopausal, and had higher proportions of infiltrating duct carcinomas with higher tumour grades, but negative lymph node status.

Detection of hormone receptor expression can guide the diagnosis and treatment for breast cancer (Zhang et al., 2013). The response to therapy depends on the IHC profile of patients as reported by previous studies (Patil et al., 2011b). Patients with ER positive breast tumors, those with ER negative but PgR positive status would be benefitted by tamoxifen; while those with HER2 positive tumors are resistant to tamoxifen (Group, 1998; Dowsett et al., 2006). Further, HER2 positive tumors respond well to targeted therapy using transtuzumab, a humanized monoclonal antibody against HER2 (Romond et al., 2005; Perez et al., 2011). The triple negative patients lack these receptors besides having larger tumor size and higher histologic grade. Thus, TNBCs patients may have relatively poorer prognosis as there are no effective systemic targeted therapies against them. We believe that a large cohort analysis would be required to assess predictive effect of these markers along with therapeutic options on the survival of breast cancer patients. Thus, comparative evaluation of the different therapeutic methods for treating TNBCs, would also be useful. The present analysis is an effort towards addressing these issues.

Our analysis also had few limitations. Inherent biases may be present due to the retrospective nature of the analysis. The database used for this analysis is not a population-based database. However, our hospital serves a large number of patients from the North Indian population and hence the results of the present analysis would be generalizable to Indian population. We could not include all patients registered in our database as shown in Figure 2. Previous studies on IHC profile of breast cancer in Indian women have reported data on small number of patients. With detailed data availability for 1284 breast cancer patients, the present analysis is one of the largest studies from India.

In conclusion, our analysis, one of the largest studies from India, provides elaborate data on IHC profile of about 1300 breast cancer patients. Majority of the Indian breast cancer patients seen in our hospital presented with ER and $\mathrm{PgR}$ positive tumors. The triple negative patients tended to be younger, premenopausal, and were associated with higher tumor grades, negative lymph nodes status and lesser frequency of ductal carcinoma in situ.

\section{Acknowledgements}

Sanofi India Limited provided financial support for data compilation, data analysis and editorial support. We would like to acknowledge the role of Dr. Ashok Krishna and Dr. Rakesh Lalchandani from Oncology Medical Affairs team of Sanofi India Limited for their scientific inputs, editorial support and coordination of Sanofi's support to this study. Dr Vidula Bhole of Episolutions Consultancy Services received consultancy fees for data analysis and editorial support from Sanofi India Limited. We would also like to clarify that Sanofi did not interfere in any aspect of the conduct of this study.

\section{References}

Ambroise M, Ghosh M, Mallikarjuna VS, et al (2011). Immunohistochemical profile of breast cancer patients at a tertiary care hospital in South India. Asian Pac J Cancer Prev, 12, 625-9.

Babu GR, Lakshmi SB, Thiyagarajan JA(2013). Epidemiological correlates of breast cancer in South India. Asian Pac J Cancer Prev, 14, 5077-83.

Badve S, Dabbs DJ, Schnitt SJ, et al (2011). Basal-like and triplenegative breast cancers: a critical review with an emphasis on the implications for pathologists and oncologists. Mod Pathol, 24, 157-67.

Basu S, Chen W, Tchou J, et al (2008). Comparison of triplenegative and estrogen receptor-positive/progesterone receptor-positive/HER2-negative breast carcinoma using quantitative fluorine-18 fluorodeoxyglucose/positron emission tomography imaging parameters: a potentially useful method for disease characterization. Cancer, 112, 995-1000.

D'Souza ND, Murthy NS, Aras RY (2013). Projection of cancer incident cases for India -till 2026. Asian Pac J Cancer Prev, 14, 4379-86.

Dogra A, Doval DC, Sardana M, et al (2014). Clinicopathological characteristics of triple negative breast cancer at a tertiary care hospital in India. Asian Pac J Cancer Prev, 15, 1057783.

Dowsett M, Houghton J, Iden C, et al (2006). Benefit from adjuvant tamoxifen therapy in primary breast cancer patients according oestrogen receptor, progesterone receptor, EGF receptor and HER2 status. Ann Oncol, 17, 818-26.

Ferlay J, Shin HR, Bray F, et al (2010). Estimates of worldwide burden of cancer in 2008: GLOBOCAN 2008. Int J Cancer, 127, 2893-917. 
Group EBCTC (1998). Tamoxifen for early breast cancer: an overview of the randomised trials. Lancet, 351, 1451-67.

J Ferlay ISI, M Ervik, R Dikshit, et al (2012). Globocan 2012: Estimated Cancer Incidence, Mortality and Prevalence Worldwide in 2012 [Online]. [Accessed 15 April 2015].

Manjunath S, Prabhu JS, Kaluve R, et al (2011). Estrogen Receptor Negative Breast Cancer in India: Do We Really Have Higher Burden of this Subtype? Indian J Surg Oncol, 2, 122-5.

Munjal K, Ambaye A, Evans MF, et al (2009). Immunohistochemical analysis of ER, PR, Her2 and CK5/6 in infiltrative breast carcinomas in Indian patients. Asian Pac J Cancer Prev, 10, 773-8.

Patil AV, Bhamre RS, Singhai R, et al (2011a). Estrogen receptor (ER) and progesterone receptor (PgR) in breast cancer of Indian women. Breast Cancer (Dove Med Press), 3, 27-33.

Patil VW, Singhai R, Patil AV, et al (2011b). Triple-negative (ER, PgR, HER-2/neu) breast cancer in Indian women. Breast Cancer (Dove Med Press), 3, 9-19.

Perez EA, Romond EH, Suman VJ, et al (2011). Four-year follow-up of trastuzumab plus adjuvant chemotherapy for operable human epidermal growth factor receptor 2-positive breast cancer: joint analysis of data from NCCTG N9831 and NSABP B-31. J Clin Oncol, 29, 3366-73.

Romond EH, Perez EA, Bryant J, et al (2005). Trastuzumab plus adjuvant chemotherapy for operable HER2-positive breast cancer. $N$ Engl J Med, 353, 1673-84.

Shet T, Agrawal A, Nadkarni M, et al (2009). Hormone receptors over the last 8 years in a cancer referral center in India: what was and what is? Indian J Pathol Microbiol, 52, 171-4.

Singhai R, Patil V, Patil A (2011). Immunohistochemical (IHC) HER-2/neu and fluorescent-in situ hybridization (FISH) gene amplification of breast cancer in Indian women. Asian Pac J Cancer Prev, 12, 179-83.

Vaidyanathan K, Kumar P, Reddy CO, et al (2010). ErbB2 expression and its association with other biological parameters of breast cancer among Indian women. Indian J Cancer, 47, 8-15.

Zhang SJ, Hu Y, Qian HL, et al (2013). Expression and significance of ER, PR, VEGF, CA15-3, CA125 and CEA in judging the prognosis of breast cancer. Asian Pac J Cancer Prev, 14, 3937-40. 